\title{
Chemical Reaction and Mass Transfer Effects on Flow of Micropolar Fluid past a Continuously Moving Porous Plate with Variable Viscosity
}

\author{
P. Loganathan ${ }^{1}$ and N. G. Stepha ${ }^{2+}$ \\ ${ }^{1}$ R.M.K Engineering College, Kavaraipettai, Chennai, 601206, India \\ ${ }^{2}$ Department of Mathematics, Anna University, Chennai 600025, India \\ †Corresponding Author Email: goldenstepha@gmail.com
}

(Received July 7, 2012; accepted October 17, 2012)

\begin{abstract}
Chemical reaction and mass transfer effects on flow of a micropolar fluid past a continuously moving porous plate with variable viscosity is investigated numerically. The plate is moving with a constant velocity in the fluid, which has a temperature dependent viscosity. The fluid viscosity is considered to vary linearly with temperature. The radiative heat flux and the viscous dissipation are taken into account in the energy equation. The partial differential equations governing the flow have been transformed into system of ordinary differential equation and solved numerically by fourth order Runge-Kutta method with shooting technique. The velocity, microrotation, temperature, concentration are shown graphically for different value of suction or injection parameter as well as temperature dependent viscosity parameter. The rate of mass transfer for different values of chemical reaction parameter and variable viscosity parameter is also shown graphically. The result shows that chemical reaction parameter and variable viscosity parameter have significant influence on heat and mass transfer rate. Effect of chemical reaction parameter and variable viscosity parameter over skin-friction coefficient and microrotation are examined.
\end{abstract}

Keywords: Microrotation, Radiation, Mass transfer, Chemical reaction, Variable viscosity.

\section{NOMENCLATURE}

$\begin{array}{ll}\mathrm{C} & \text { species concentration } \\ \mathrm{C}_{\mathrm{p}} & \text { specific heat } \\ \mathrm{D} & \text { binary diffusion coefficient } \\ \mathrm{Ec} & \text { Eckert number } \\ \mathrm{FW} & \text { suction or injection parameter } \\ \mathrm{f} & \text { dimensionless stream function } \\ \mathrm{G} & \text { micro-rotation parameter } \\ \mathrm{g} & \text { dimensionless micro rotation } \\ \mathrm{K} 1 & \text { coupling constant } \\ k^{\prime} & \text { mean absorption coefficient } \\ \mathrm{N} & \text { radiation parameter } \\ \mathrm{Pr} & \text { Prandtl number } \\ \mathrm{qr} & \text { radio active heat flux } \\ \mathrm{R} & \text { chemical reaction parameter } \\ \mathrm{Sc} & \text { Schmidt number }\end{array}$

$\begin{array}{ll}\mathrm{T} & \text { temperature } \\ \mathrm{U}_{0} & \text { velocity of the plate } \\ \mathrm{u} & \text { velocity in x direction } \\ \mathrm{v} & \text { velocity in y direction } \\ \mathrm{x} & \text { distance along the surface } \\ \mathrm{y} & \text { distance normal to the surface } \\ \varphi & \text { dimensionless concentration } \\ \gamma_{s} & \text { spin gradient viscosity } \\ \eta & \text { similarity Variables } \\ \mu_{f} & \text { reference viscosity } \\ \theta & \text { dimensionless temperature } \\ \sigma & \text { micro-rotation component } \\ \sigma_{1} & \text { Stefan-Boltzmann constant } \\ \text { Subscript w } & \text { condition on the wall } \\ \infty & \text { free stream condition }\end{array}$




\section{INTRODUCTION}

The boundary layer flow of micropolar fluid has received considerable attention for the past few years, especially after the excellent work of Eringen (1964). Micropolar fluid is one of the complex fluids with microstructure. It consists of rigid, randomly oriented particles suspended in a viscous medium. Due to the rotation of the particle, the governing equation of the flow contains microrotation field in addition to velocity field, so it can exhibit the effect of microrotation occurred in micropolar fluid such as liquid crystals, animal fluid, and some polymeric fluids accurately. Eringen (1972) extended this theory to thermomicropolar theory. The problem of flow of Newtonian fluid past continuously moving plate was introduced by Sakiadis (1961) and similarity transformation is used to determine the numerical solution. Heat transfer effect on a continuously moving plate is analyzed by Tsou et al. (1967) and it showed that this flow is physically attainable under laboratory condition. Ebert (1973) revealed that under comparable flow condition, polar fluid would exhibit a greater resistance than a Newtonian fluid. The excellent review on micropolar fluid was given by Ariman et al. (1974).

Due to many engineering process such as extrusion of plastic sheet, crystal growing, polymer sheet extruded continuously for a die etc., the boundary layer flow of micropolar fluid on a continuously moving surface becomes an important area of research. Soundalgekar and Takhar (1983) studied the heat transfer of micropolar fluid past continuously moving plate by considering the fluid medium at rest for constant microinertia. The knowledge of radiation effect plays a vital role in nuclear power plant, gas turbines, and space vehicles. Heat transfer of a micropolar fluid in the presence of radiation was analyzed by Perdikis and Raptis (1996). Raptis (1998) reported the effect of radiation on flow of a micropolar fluid past a continuously moving plate and as a result increasing radiation parameter has the effect of decreasing the temperature. Hassan and Arabawy (2003) studied radiation effect on the flow of a micropolar fluid past a continuously moving plate with suction/injection. Anjalidevi and Ganga (2010) performed the effect of viscous dissipation on MHD nonlinear flow and heat transfer past a porous medium with prescribed heat flux. Govardhan and Kishan (2012) focused the unsteady MHD boundary layer flow of an incompressible micropolar fluid over a stretching sheet.

Combined heat and mass transfer effect in moving fluid is also important in view of several physical problems. Heat and mass transfer occur in the process such as drying evaporation at the surface of water body, energy transfer in wet cooling tower and the flow in a desert cooler simultaneously. Recently Loganathan and Golden stepha (2012) studied the problem of heat and mass transfer effects on micropolar fluid past continuously moving flat plate in the presence of radiation. The study of combined heat and mass transfer problems with chemical reaction are also important in many processes such as heat exchangers that are used for the cooling of electronic circuits, packed bed chemical reactor, and also in radioactive waste georepositories. In many chemical processes, chemical reaction takes place between the surface and the fluid, which moves due to continuous movement of the surface. Chemical reaction can be classified into homogeneous and heterogeneous. If the rate of reaction is directly proportional to spices concentration, then it is said to be first order chemical reaction. Recently Anand Rao et al. (2012) studied the Chemical reaction effects on an unsteady MHD free Convection fluid flow past a semi-infinite vertical plate Embedded in a porous medium with heat absorption.

Most of the studies on heat and mass transfer are with constant properties; however, it is known that physical properties vary considerably with temperature. For example in lubricant fluid, raise in temperature due to internal friction affects the viscosity, so the viscosity is no longer constant. It is essential to consider viscosity as variable quantity to predict the flow behavior accurately. Moreover, Kabeir (2004) studied the effect of radiation and heat transfer on micropolar fluid with variable viscosity.

In literature survey, so far no research has been made to analyze the problem of chemical reaction and mass transfer effects on flow of micropolar fluid past a continuously moving porous plate with variable viscosity. Hence, the objective of the present paper is to study the effects of variable viscosity and chemical reaction on a micropolar fluid past continuously moving porous plate with radiation by considering the mass diffusion process simultaneously for all aspects of the flow.

\section{Formulation of a Problem}

Consider a steady, two-dimensional incompressible heat and mass transfer flow on a continuously moving flat porous plate with a constant velocity with variable viscosity in a micropolar fluid medium at rest as shown in Fig 1. The origin of the coordinate system is placed at the place where the plate is drawn into the fluid medium (slot).

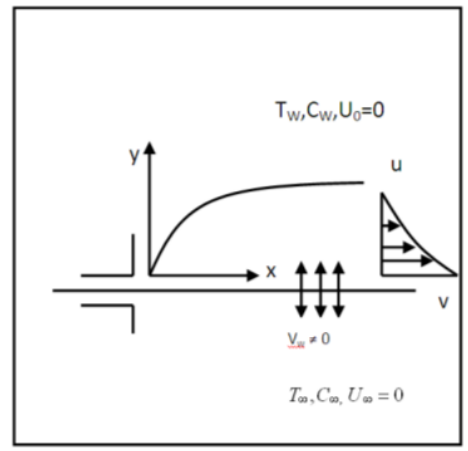

Fig. 1. Coordinate system and flow model

The coordinate axis $\mathrm{x}$ is taken along the plate and $\mathrm{y}$-axis is normal to it. The surface of the plate is maintained at a uniform temperature $T_{W}$ and a uniform concentration $C_{W}$. The fluid viscosity is considered to 
vary linearly with temperature as given below $\mu=\mu_{f}\left(1+\gamma_{f}(\theta-1 / 2)\right)$. Viscous dissipation in the energy equation is also taken in to account.

Under the above assumption, the boundary layer governing the flow, angular velocity, and heat transfer of a micropolar fluid on a continuously moving plate are given by Raptis, (1998) and Arabawy, (2003). In addition to that, species diffusion equation is considered to analyze the mass transfer effect.

$\frac{\partial u}{\partial x}+\frac{\partial v}{\partial y}=0$

$u \frac{\partial u}{\partial x}+v \frac{\partial u}{\partial y}=\frac{1}{\rho} \frac{\partial}{\partial y}(\mu+s) \frac{\partial u}{\partial y}+k_{1} \frac{\partial \sigma}{\partial y}$

$\gamma_{s} \frac{\partial^{2} \sigma}{\partial y^{2}}-2 \sigma-\frac{\partial u}{\partial y}=0$

$u \frac{\partial T}{\partial x}+v \frac{\partial T}{\partial y}=\frac{k}{\rho c_{p}}\left(\frac{\partial^{2} T}{\partial y^{2}}\right)+\frac{\gamma}{c_{p}}\left(\frac{\partial u}{\partial y}\right)^{2}-\frac{1}{\rho c_{p}} \frac{\partial q_{r}}{\partial y}$

$u \frac{\partial C}{\partial x}+v \frac{\partial C}{\partial y}=D \frac{\partial^{2} C}{\partial y^{2}}-R_{C}\left(C-C_{\infty}\right)$

With boundary condition

$u=U_{0}, \quad v=V_{W}, T=T_{W}, \quad \sigma=0$, at $y=0$

$u=0, \quad v=0, \quad T=T_{\infty}, \quad \sigma=0$, as $y \rightarrow \infty$

and the boundary conditions for diffusion Eq. (5) are $C=C_{W}$ at $y=0, C=C_{\infty}$, as $y \rightarrow \infty$ where $\mu$ is the dynamic viscosity, $\mathrm{s}$ is the gyro viscosity, $\rho$ is the density of the fluid, $k_{1}=\frac{s}{\rho}$ coupling constant, $U_{0}$ is the uniform velocity of the plate, $V_{W}$ is the non zero velocity component of the wall.

The rosseland approximation (Loganathan and Ganesan, 2002) is used to describe the radiative heat flux in the energy equation, which leads to the radiative heat flux $q_{r}=-\frac{4 \sigma_{1}}{3 k^{\prime}} \frac{\partial T^{4}}{\partial y}$ where $\sigma_{1}$ is the Stefan

Boltzmann constant and $k^{\prime}$ is the mean absorption coefficient. If the temperature difference with in the flow are sufficiently small such that $T^{4}$ may be expressed as linear function of the temperature, then the Taylor's series $T^{4}$ about $T_{\infty}$, after neglecting the higher order terms is given by

$T^{4} \approx 4 T_{\infty}^{3} T-3 T_{\infty}^{4}$

In view of Eq. (6) and Eq. (7), Eq. (4) becomes

$u \frac{\partial T}{\partial x}+v \frac{\partial T}{\partial y}=\frac{k}{\rho c_{p}} \frac{\partial^{2} T}{\partial y^{2}}+\frac{\gamma}{c_{p}}\left(\frac{\partial u}{\partial y}\right)^{2}+\frac{16 \sigma_{1} T_{\infty}^{3}}{3 K^{\prime} \rho c_{p}}\left(\frac{\partial^{2} T}{\partial y^{2}}\right)$

Now considered the following dimensionless similarity transform

$\eta=y \sqrt{\frac{U_{0}}{2 \gamma x}}, \psi=\sqrt{2 \gamma U_{0} x} f(\eta), \sigma=\sqrt{\frac{U_{0}^{3}}{2 \gamma x}} g(\eta)$,

$$
\begin{aligned}
& \theta=\frac{T-T_{\infty}}{T_{W}-T_{\infty}}, \quad \varphi=\frac{C-C_{\infty}}{C_{W}-C_{\infty}}, K=\frac{k_{1}}{\gamma}, \quad G=\frac{U_{0} \gamma_{s}}{\gamma x}, \\
& \operatorname{Pr}=\frac{\gamma \rho c_{\rho}}{k}, \quad N=\frac{k k}{4 \sigma_{1} T_{\infty}^{3}}, E c=\frac{U_{0}^{2}}{c_{p}\left(T_{W}-T_{\infty}\right)}, \\
& R=-R_{C}\left(\frac{2 x}{U_{0}}\right), F_{W}=-V_{W} \sqrt{\frac{2 x}{\gamma U_{0}}}
\end{aligned}
$$

where $f(\eta), g(\eta)$ are dimensionless stream functions. In view of Eq. (9), Eqs. (2)- (5) and Eq.(8) are reduced to the following ordinary differential equations

$$
\begin{aligned}
& \left(1+\gamma_{f}(\theta-0.5)+k\right) f^{\prime \prime \prime}+f f^{\prime \prime}+\frac{k}{2} g^{\prime}+\gamma_{f} \theta^{\prime} f^{\prime \prime}=0 \\
& G g^{\prime \prime}-4 g-2 f^{\prime \prime}=0 \\
& (3 N+4) \theta^{\prime \prime}+3 \operatorname{Pr} N \theta^{\prime}+3 N \operatorname{Pr} E c\left(f^{\prime \prime}\right)^{2}=0 \\
& \varphi^{\prime \prime}+S c f \varphi^{\prime}+S c R \varphi=0
\end{aligned}
$$

The corresponding initial and boundary conditions in non-dimensional quantities are given by

$$
\begin{aligned}
& \eta=0: f(0)=F_{W}, f^{\prime}(0)=1, \theta(0)=1, g(0)=0, \varphi(0)=1 \\
& \eta=\infty: f^{\prime}(\infty)=0, \theta(\infty)=0, g(\infty)=0, \varphi(\infty)=0
\end{aligned}
$$

The parameters G, R, A, and FW correspond to local effects, that is, relating to specific values of $\mathrm{x}$. Many authors including Raptis (1998), Arabawy (2003), Rahman and Sattar (2007), Rahman (2009), Rahman et al. (2009), M.S. Alam et al. (2009), Kabeir (2004) are used the above similarity analysis and the same has been used in the present problem. Therefore Eq. (10) to Eq. (13) are ordinary differential equations, which are locally similar together with the boundary conditions Eq. (14).

The interesting physical parameters are local skinfriction coefficient and the local Nusselt number and local Sherwood number which can be defined as follows $C_{f}=\frac{2 \tau_{W}}{\rho U_{0}^{2}}, N u_{x}=\frac{x q_{W}}{k\left(T_{W}-T_{\infty}\right)}, S h_{x}=\frac{x q_{M}}{D\left(C_{W}-C_{\infty}\right)}$ where

$\tau_{W}=\left[(\mu+K) \frac{\partial u}{\partial y}+K \sigma\right]_{y=0}, q_{W}(x)=-k\left(\frac{\partial T}{\partial y}\right)_{y=0}, q_{M}(x)=-D\left(\frac{\partial C}{\partial y}\right)_{y=0}$

Using similarity variable Eq. (9), we get

$C_{f}=-2 \operatorname{Re}_{x}^{-1 / 2} f^{\prime \prime}(0), \quad N u_{x}=\frac{-\operatorname{Re}_{x}^{1 / 2} \theta^{\prime}(0)}{\sqrt{2}}, \quad S h_{x}=\frac{-\operatorname{Re}_{x}^{1 / 2} \varphi^{\prime}(0)}{\sqrt{2}}$.

\section{NUMERICAL SOLUTION}

The coupled non-linear ordinary differential equations Eqs. (10), (11), (12), and (13) with the boundary condition Eq. (14) are solved numerically by fourth order Runge-Kutta method along with NactsheimSwigert shooting technique (Adams and Rogers, 1973) for the prescribed parameter $\mathrm{F}_{\mathrm{w}}, \mathrm{K}, \mathrm{N}, \mathrm{Pr}, \mathrm{Sc}, \mathrm{G}, \mathrm{R}$, and $\gamma_{f}$. In the boundary condition Eq. (14), there are four asymptotic boundary condition and hence there are four 
unknown surface condition $f^{\prime \prime}(0), g^{\prime}(0), \theta^{\prime}(0)$, and $\varphi^{\prime}(0)$. Values of these unknown surface conditions are obtained by Nactsheim-Swigert technique (Adams and Rogers, 1973). A computer program was set up for the above- mentioned procedure along with fourth order Runge-Kutta method to solve the Eqs. (10) - (13) with boundary condition Eq. (14). A step size of $\Delta \eta=0.01$ was selected to satisfy the convergence criterion of $10-4$ in all cases.

\section{RESULT AND DISCUSSION}

To discuss the effect of the variable viscosity $\gamma_{f}$, chemical reaction $\mathrm{R}$, and suction or injection parameter $F_{w}$, the numerical solution is given as velocity, temperature, concentration, and angular momentum profiles for physical parameters such as $\mathrm{G}, \mathrm{K}, \mathrm{N}, \mathrm{Sc}$, $\mathrm{R}, \gamma_{f}$ and $\operatorname{Pr}$. The present value of heat transfer parameter $-\theta^{\prime}(0)$ is also compared with Arabawy (2003) and it has excellent agreement with them.

The dimensionless velocity component for different values of suction or injection parameter $F_{w}$ and chemical reaction parameter $\mathrm{R}$ with $\mathrm{K}=0.2, \mathrm{~N}=5.0$, $\mathrm{Sc}=2.0, \gamma_{f}=0.8$ and $\operatorname{Pr}=0.733$ is portrayed in Fig. 2 . It is observed that for increasing value of $F_{w}$, the velocity field gradually decreases. If $F_{w}>0$ then the resistance of the fluid increases and has a tendency to reduce the velocity of the flow, but wall injection $\left(\mathrm{F}_{\mathrm{w}}<\right.$ $0)$ produces the opposite effect. This behavior is clearly seen from Fig. 2. Also it is observed that change in chemical reaction parameter has no effect on the velocity profile.

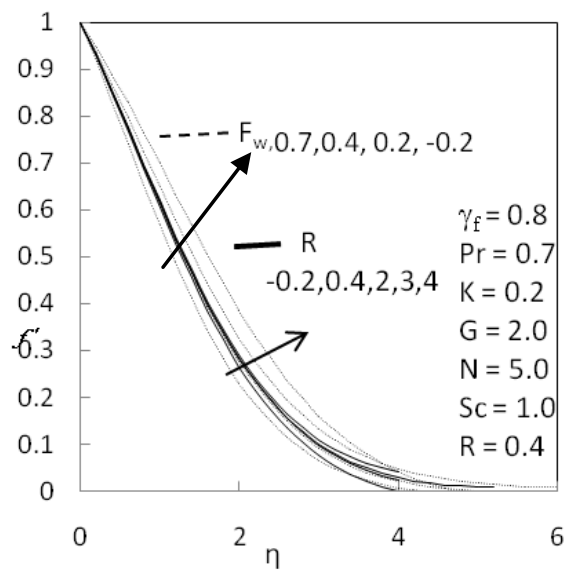

Fig. 2. Velocity profile for different values of suction and injection and reaction parameter

Angular velocity profiles for various $\mathrm{F}_{\mathrm{w}}$ are presented in the Fig. 3. It may be noticed that for $F_{w}<0$, angular velocity profiles increases within the domain $\eta<1.0$ with increasing value of $F_{w}$. This is because of the rotation of the micro constituents induced near the surface of the plate due to increase of suction velocity. Outside this region, kinematic viscosity is dominant, so angular velocity profiles overlap and decrease with increasing value of $\mathrm{F}_{\mathrm{w}}$. It is interesting to notice that if $\mathrm{F}_{\mathrm{w}}$ is less, then it takes more time to reach its maximum, however, the exact opposite behavior takes place for increasing value for $\mathrm{F}_{\mathrm{w}}$. It is observed that for $\mathrm{F}_{\mathrm{w}}=0.0$, the angular velocity profile reaches the maximum at $\eta=1.5$ and for $\mathrm{F}_{\mathrm{w}}=0.7$, the angular velocity profile reaches the maximum at $\eta=0.8$.

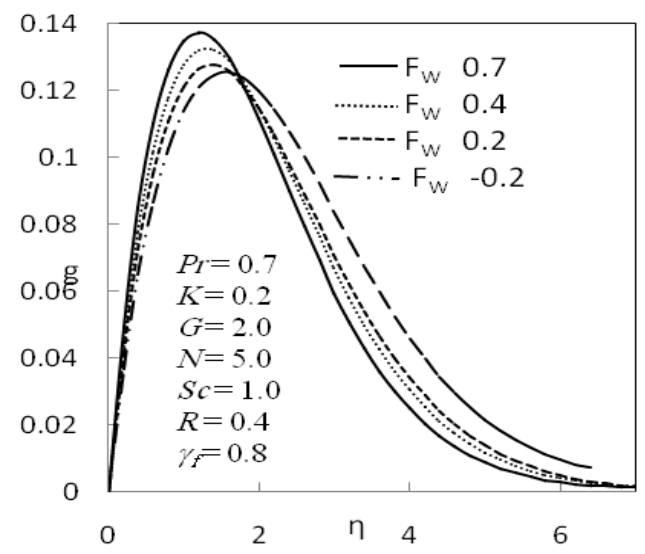

Fig. 3. Micro rotation profile for different values of suction and injection

The effects of suction or injection parameter $F_{w}$ on the temperature profile for the value of $\mathrm{Pr}=0.733$ and $\mathrm{Pr}=$ 7 are shown in Fig. 4. Similar to the velocity field, wall suction $\left(F_{w}>0\right)$ has a tendency to reduce the thermal boundary layer thickness and wall injection $\left(F_{w}<0\right)$ has a tendency to increase the thermal boundary layer thickness. Because of this fact, the temperature field gradually decreases with increasing value of $F_{w}$ in both these cases.

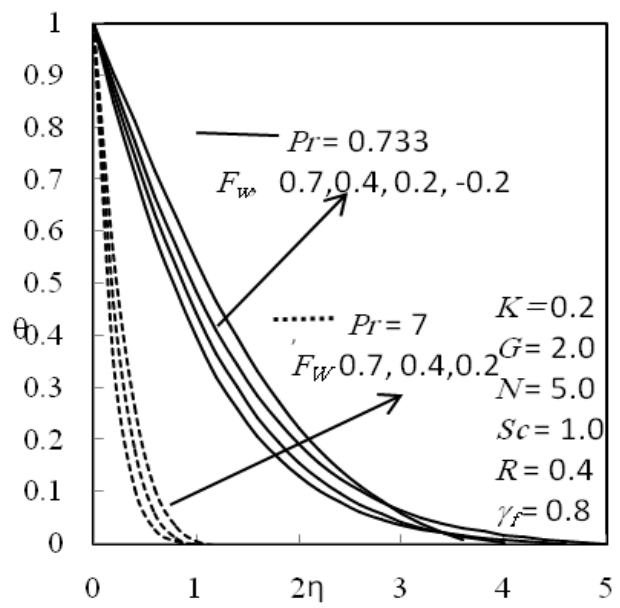

Fig. 4. Temperature profile for different values of suction and injection

The effects of suction or injection parameter $F_{w}$ on concentration profile for $\mathrm{Sc}=1.0, \operatorname{Pr}=7.0, \mathrm{~K}=0.2, \mathrm{G}$ $=2.0, \gamma_{f}=0.8$ and $\mathrm{N}=5.0$ are shown graphically in Fig. 5. It is observed that for increasing value of $F_{w}$, the concentration profile decreases. The concentration profile for different values of $\mathrm{R}, \mathrm{Pr}=0.7, \mathrm{~K}=0.2, \mathrm{G}=$ 2.0, $\gamma_{f}=0.8, \mathrm{~N}=5.0$, and $\mathrm{Sc}=1.0$ are shown in Fig. 6 . It is observed that the increasing value of $\mathrm{R}$ the concentration profile decreases. Moreover, smaller values of $\mathrm{R}$, concentration profile gradually decreases, whereas large values of $R$ affect the concentration profile tremendously. It can be observed that the 
concentration changes positive to negative and then reaches to zero. This is because of concentration of the fluid does not remains constant and is consumed continuously during the course of reaction, so the concentration decreases as $\mathrm{R}$ increases. The temperature profile for different values of $\mathrm{Pr}$ and $\mathrm{R}, \mathrm{N}=5.0 \mathrm{Sc}=1.0$, $\gamma_{f}=0.8$, and $\mathrm{F}_{\mathrm{w}}=0.7$ are shown in Fig. 7. It shows that the temperature decreases with increasing value of Pr. For smaller value of Pr, thermal conduction is more, so the heat is able to diffuse more rapidly than higher values of $\operatorname{Pr}$ and also move away from the heat surface quickly than higher values of Pr. It is also observed that change in chemical reaction parameter has no effect on the temperature profile.

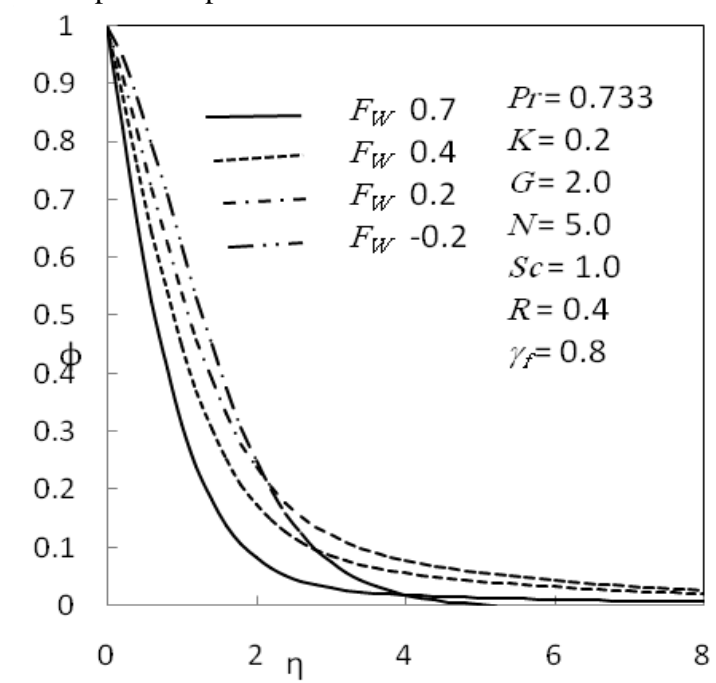

Fig. 5. Concentration profile for different values of suction and injection

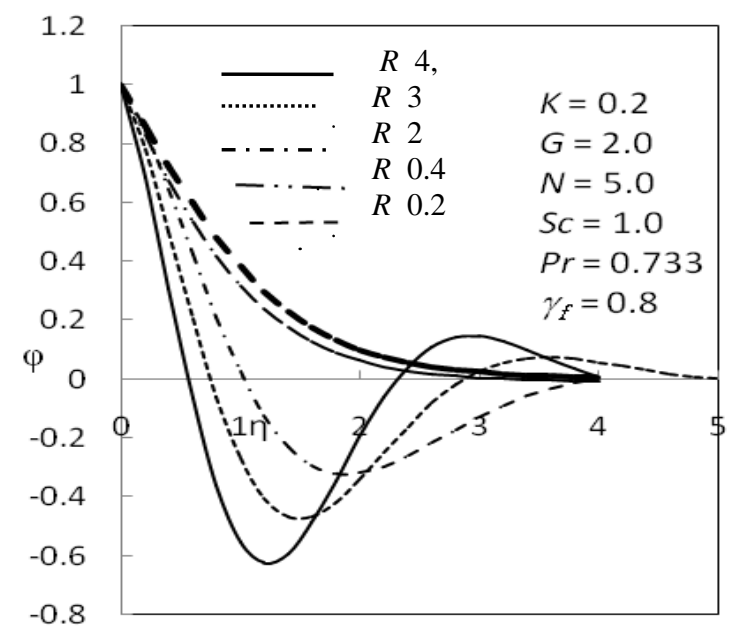

Fig. 6. Concentration profile for different values of $\mathrm{R}$

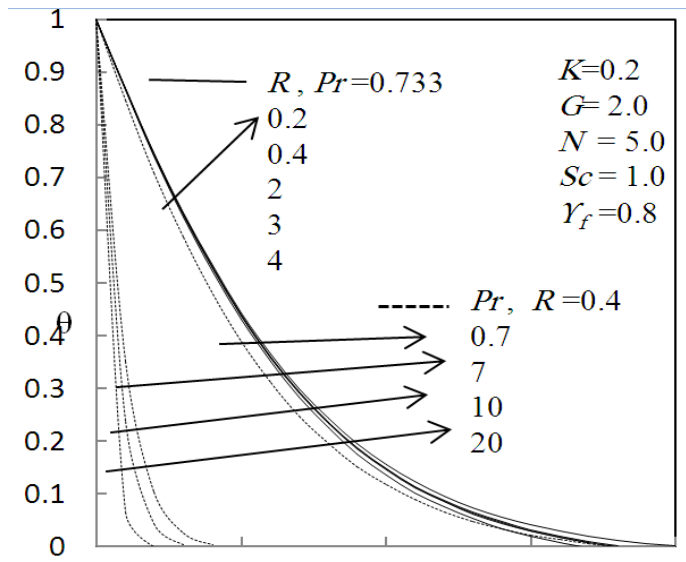

Fig. 7. Temperature profile for different values of $\mathrm{R}$ and different values of $\mathrm{Pr}$

Figures 8 to 11 display the velocity, temperature, concentration, and angular momentum profiles for different values of $\gamma_{f}$. It shows that the velocity profile increases with increasing value of temperaturedependent fluid viscosity parameter $\gamma_{f}$, but the temperature and concentration decreases with increasing value of $\gamma_{f}$. In Fig. 8, the velocity is found to be increase with the increase of temperaturedependent viscosity parameter. This increasing value of $\gamma_{f}$ causes decrease in fluid viscosity, which results the increment in the velocity and the boundary layer thickness. With an increase of $\gamma_{f}$, fluid velocity increases whereas the fluid temperature decreases. In Fig. 10, the temperature is found to decrease with increasing value of $\gamma_{f}$ as expected. The concentration profile for different values of $\mathrm{Sc}, \mathrm{Pr}=0.733, \mathrm{~K}=0.2, \mathrm{G}$ $=2.0$ and $\mathrm{N}=5.0$ are also shown in Fig. 11. It is also observed that with the increasing value of $\mathrm{Sc}$ the concentration profile decreases. Increasing Sc indicates that the species diffusion reduces and viscous force increases which cause a decrease in concentration as expected.

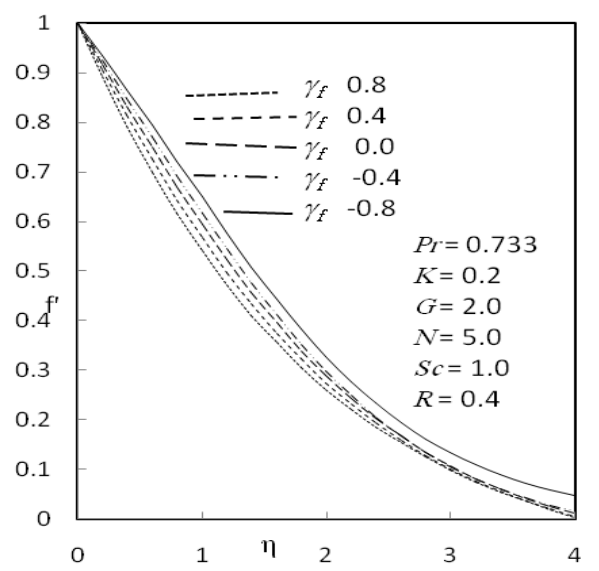

Fig. 8. Velocity profile for different variable viscosity 
P. Loganathan and N. G. Stepha / JAFM, Vol. 6, No. 4, pp. 581-588, 2013.

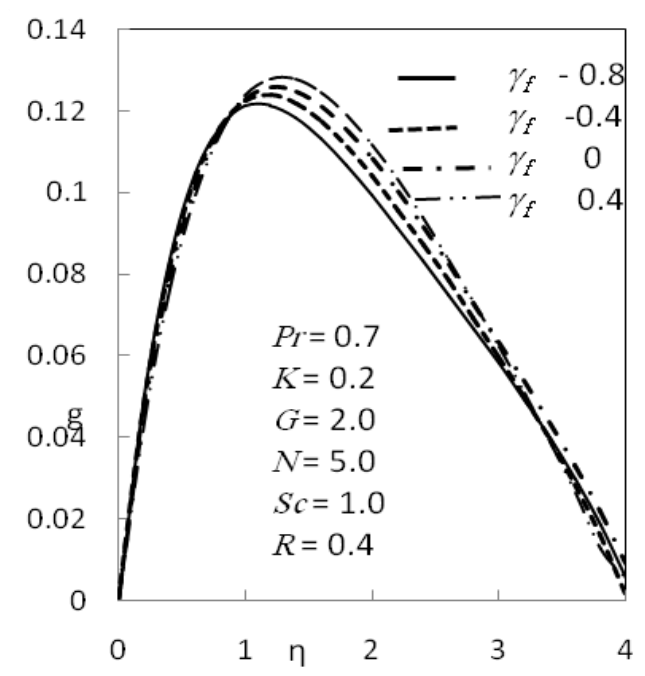

Fig. 9. Microrotation profile for different variable viscosity

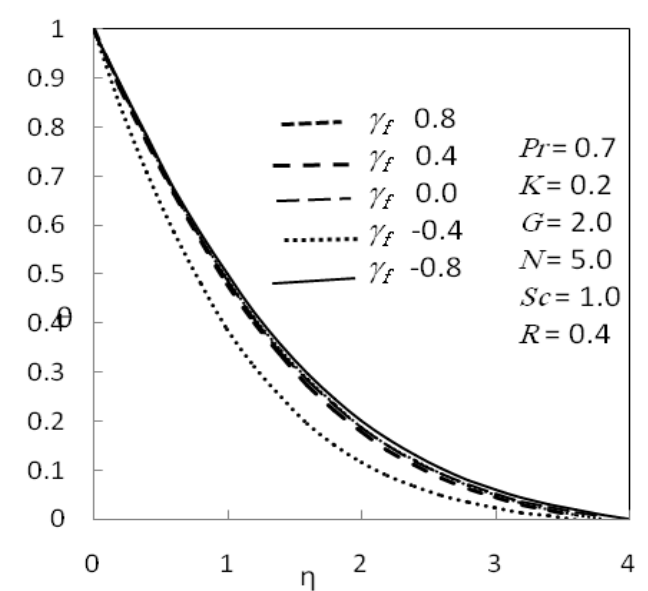

Fig. 10. Temperature profile for different viscosity

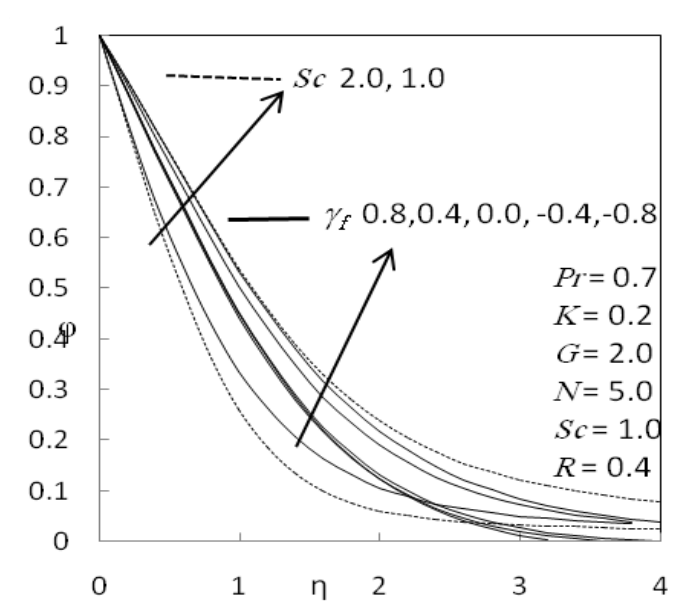

Fig. 11. Concentration profile for different viscosity and for different values of Sc

Figure 12 depicts the effects of $\operatorname{Pr}$ on the heat transfer for various values of suction or injection parameter $F_{w}$. It is observed that heat transfer increase quite rapidly with increasing Pr. Figure 13 shows the variation in skin-friction coefficient against the suction or injection parameter $\mathrm{F}_{\mathrm{w}}$ for different values of $\gamma_{f}$.

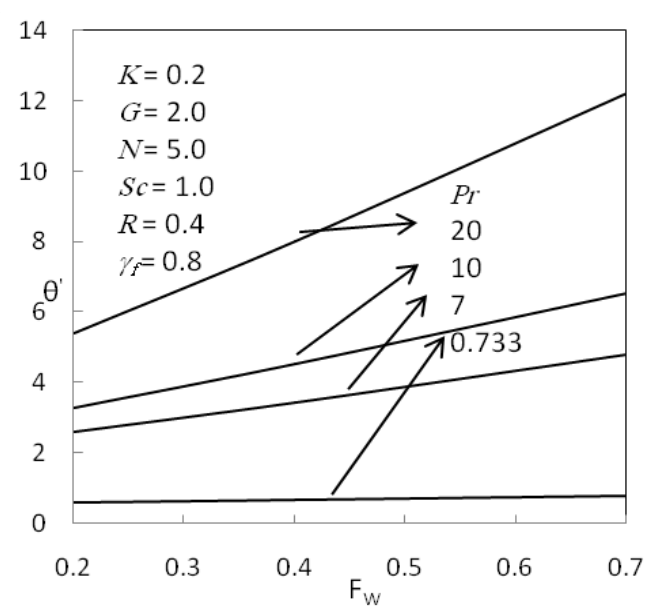

Fig. 12. Heat transfer parameter for different values of $\operatorname{Pr}$

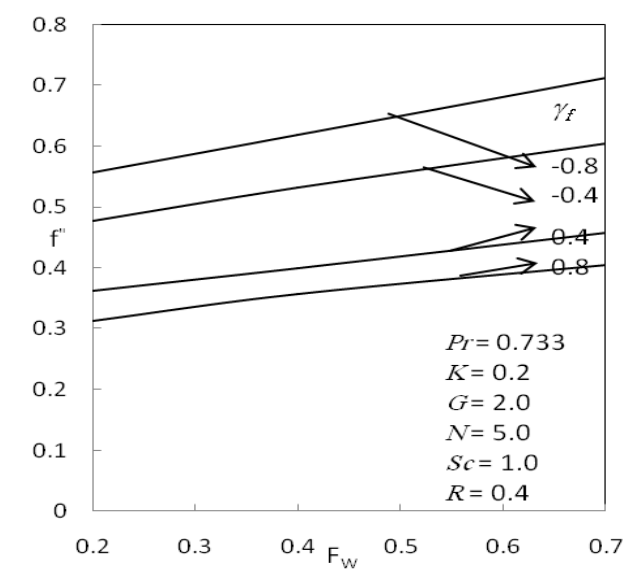

Fig.13. Skin-friction coefficient against suction parameter for different value of viscosity

Figure 14 shows the variation in microrotation coefficient against the suction or injection parameter $\mathrm{F}_{\mathrm{w}}$ for different values of $\gamma_{f}$. From Fig. 13 and Fig. 14, it can be seen that skin-friction coefficient and microrotation coefficient decreases for increasing value of $\gamma_{f}$.

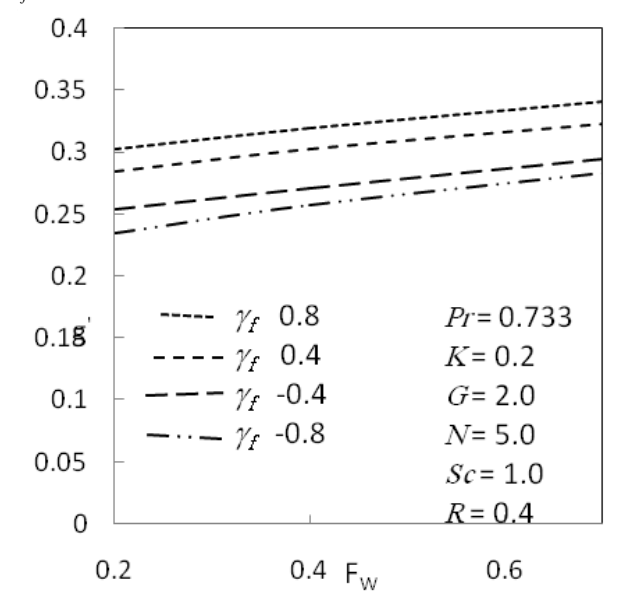

Fig. 14. Microrotation parameter against suction parameter for different value of viscosity 
Figure 15 shows the variation in rate of mass transfer against the suction or injection parameter $F_{w}$ for different values of $\gamma_{f}$. The effects of chemical reaction parameter $\mathrm{R}$ on the mass transfer for various values of suction or injection parameter $F_{w}$ are also plotted in Fig. 15. It is observed that the rate of mass transfer decreases for increasing value of $\mathrm{R}$, but it increases for increasing value of $\gamma_{f}$.

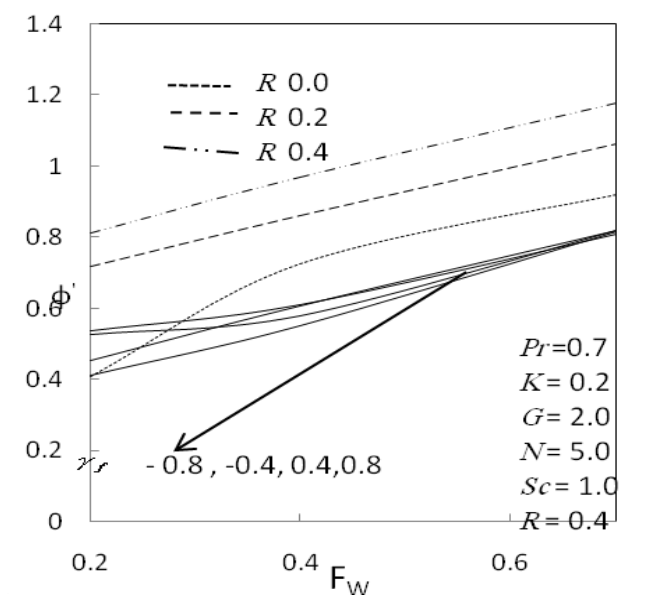

Fig. 15. Rate of mass transfer against suction parameter for different value of viscosity and chemical reaction parameter

\section{CONCLUSION}

A numerical study has been carried out to study the effect of radiation on the flow of a micropolar fluid past continuously moving plate in the presence of mass transfer. The governing equations are transformed into system of non-linear ordinary differential equations by using similarity variables. It is solved numerically by using fourth order Runge-Kutta method along with Nactsheim-Swigert shooting technique (Adams and Rogers, 1973). Computation is carried out for the prescribed parameter $\mathrm{F}_{\mathrm{w}}, \mathrm{K}, \mathrm{N}, \mathrm{Pr}, \mathrm{Sc}, \mathrm{R}, \gamma_{f}$, and $\mathrm{G}$.

Conclusions of this study are as follows;

(i) The velocity, temperature, concentration profiles decreases when the suction or injection parameter $\mathrm{F}_{\mathrm{w}}$ increases.

(ii) Also the temperature decreases due to increase in Prandtl number and concentration profile decreases at the increase of Sc.

(iii) For increasing value of $\mathrm{R}$ the concentration profile decreases. It is also observed that for smaller values $\mathrm{R}$, concentration profile gradually decreases, and for large values $\mathrm{R}$ concentration profile changes positive to negative and then reaches to zero.

(iv) For increasing value of temperature-dependent viscosity parameter, the temperature and concentration profile decreases.

(v) The velocity profile increases for increasing value of temperature-dependent viscosity parameter. (vi) The heat transfer parameter increases as the increasing value of $\mathrm{Pr}$.

(vii) The influences of chemical reaction parameter and temperature-dependent viscosity parameter on mass transfer are shown in the figures.

For increasing temperature-dependent viscosity, skinfriction coefficient and microrotation parameter decreases.

Table 1 Comparison value of $-\theta^{\prime}(0)$ for various values of $\operatorname{Pr}$ and $\mathrm{K}=0.2, \mathrm{G}=2, \mathrm{~N}=5.0, \mathrm{Ec}=$ $0.02, \mathrm{~F}_{\mathrm{W}}=0, \mathrm{R}=0, \mathrm{~A}=0, \gamma_{f}=0.0$

\begin{tabular}{|c|c|c|}
\hline Pr & Arabawy (2003) & Present work \\
\hline 0.73 & 0.4270 & 0.508150 \\
7 & 1.6986 & 1.77500 \\
10 & 2.06029 & 2.139328 \\
20 & 2.9719 & 3.061166 \\
\hline
\end{tabular}

\section{REFERENCES}

Adams, J.A. and D.F. Rogers (1973). ComputerAided Heat Transfer Analysis, McGraw-Hill.

Ahmadi G., (1976). "Self-similar solution of incompressible micropolar boundary layer flow over semi infinite plate", Int. J. Eng. Sci., 14, $639-646$.

Alam, M.S., M.M. Rahman and M.A. Sattar (2009). On the effectiveness of viscous dissipation and joule heating on steady magnetohydrodynamic heat and mass transfer flow over an inclined radiate isothermal permeable surface in the presence of thermophoresis, Commun. Nonlinear Sci. Numer. Simul, 14, 2132-2143.

Anand Rao, J., S. Sivaiah and R. Srinivasa Raju (2012). Chemical reaction effects on an unsteady MHD free Convection fluid flow past a semi-infinite vertical plate Embedded in a porous medium with heat absorption, Journal of Applied Fluid Mechanics, 5(3), 63-70.

Anjalidevi, S.P and B. Ganga (2010). Dissipation effects on MHD nonlinear flow and heat transfer past a porous medium with prescribed heat flux, Journal of Applied Fluid Mechanics, 13(5), 1-6.

Arabawy, H. A. M. El. (2003). Effect of suction/injection on the flow of a micropolar fluid past a continuously moving plate in the presence of radiation, Int. J. Heat Mass Transfer, 46, 14711477.

Ariman, T., M.A. Turk and N.D. Sylvester (1974). Application of Microcontinuum fluid mechanics. Int. J. Eng. Sci., 12, $273-293$. 
P. Loganathan and N. G. Stepha / JAFM, Vol. 6, No. 4, pp. 581-588, 2013.

Ebert, F. (1973). A Similarity solution for the boundary layer flow of a polar fluid, Chemical Eng. Journal, 5, 72-85.

El-Kabeir, S.M.M, (2004). Radiative effect on forced convection flows in micropolar fluids with variable viscosity, Can.J.Phys., 82, 151-165.

Eringen, A.C. (1964). Simple Microfluids, Int. J. Eng. Sci., 2, 205 - 207.

Eringen, A.C. (1972). Theory of Thermomicrofluids, Journal of Mathematical Analysis and Application, 38, 480-496.

Ganesan, P. and P. Loganathan (2002). Radiation and mass transfer effects on flow of an incompressible viscous fluid past a moving vertical cylinder, Int. J. Heat Mass Transfer, 45, 4281 - 4288.

Govardhan, K and N. Kishan (2012). Unsteady MHD Boundary Layer Flow of an Incompressible Micropolar Fluid over a Stretching Sheet, Journal of Applied Fluid Mechanics, 5(3), 23-28.

Loganathan, P. and N. Golden Stepha (2012). Radiation and mass transfer effects on flow of micropolar fluid past a continuously moving plate with suction/injection, Int .Review of Mechanical Engineering, 6(1).
Perdikis, C. and A. Raptis (1996). Heat transfer of a micropolar fluid by the presence of radiation, Heat Mass Transfer, 31, 381- 382.

Rahman, M. M. (2009). Convective flows of micropolar fluids from radiate isothermal porous surfaces with viscous dissipation and joule heating, Commun. Nonlinear Sci. Numer. Simul. 14, 3018-3030.

Rahman, M. M. and M. A. Sattar (2007). Transient convective flow of micropolar fluid past a continuously moving vertical porous plate in the presence of radiation, Int. J. App. Mech. Eng., $12,497-513$.

Raptis, A. (1998). Flow of a micropolar fluid past continuously moving plate by the presence of radiation, Int. J. Heat Mass Transfer, 4, 28652866.

Sakiadis, B.C. (1961). Boundary-layer behavior on continuous solid surfaces: I. The Boundary layer on a continuous flat surface, AIChE J, 7, 221225.

Soundalgekar, V.M. and H.S. Takhar (1983). Flow of a micropolar fluid on a continuous moving plate, Int. J. Eng. Sci., 21, 961-965.

Tsou, F., E. Sparrow and R. Goldstein (1967). Flow and heat transfer in the boundary layer on a continuous moving plate, Int. J. Heat Mass Transfer, 10, 219- 235. 